\title{
Electromechanical Performance Comparison for Different CMUT Element Geometries
}

\author{
Jorge Mendoza-López \\ Instituto de Microelectrónica de Sevilla \\ c/ Américo Vespucio s/n \\ Sevilla, Spain \\ mendoza@imse-cnm.csic.es
}

\author{
Carlos Sánchez-López \\ Universidad Autónoma de Tlaxcala \\ Av. Apizaquito km 1.5 \\ Apizaco-Tlaxcala, 70300 Mexico \\ carlsanmx@yahoo.com.mx
}

\begin{abstract}
Different capacitive micromachined ultrasonic transducers (CMUTs) element geometries and fabrication techniques have been proposed through the years though the questions of which element geometry suits each application best as well as further geometrical, array and design parameter optimization techniques still remain open. This paper proposes a thorough comparison between square, hexagonal and circular CMUT elements and geometries through finite element method (FEM) simulations and provides results comparing their respective electromechanical parameters.
\end{abstract}

Keywords- CMUT; MEMS; FEM; geometry comparison; capacitive micro ultrasonic transducer; medical ultrasound; nondestructive evaluation; NDT

\section{INTRODUCTION}

Capacitive micro ultrasonic transducers (CMUTs) already constitute the current generation of ultrasound imaging transducers for biomedical applications and nondestructive evaluation tests. CMUTs currently constitute an economic and viable alternative for non-destructive testing (NDT) and medical ultrasound applications, as opposed to traditional piezoelectric ceramic transducers. CMUTs started to be developed in the early 2000 [1] and emerged as a promising alternative for ultrasound transduction.

The traditionally big piezoelectric crystals with which transducer arrays were made have by now largely been replaced by micro electromechanical systems (MEMS) capacitive or piezoelectric devices, which offer a smaller alternative, array capabilities, device characteristic lengths of less than $50 \mu \mathrm{m}$ and square matrix arrays of 256 elements which can be contained in less than $0.25 \mathrm{~cm}^{2}$. At the same time, due to their reduced size, CMUTs offer a larger bandwidth than their counter piezoelectric macroscopic ultrasound transducers, as well as a potential for integration with CMOS electronics, easiness to fabricate large arrays, plus a reasonably good coupling coefficient to the medium. This last factor can be modified adding extra matching layers to the MEMS fabricated devices. The small size capability of CMUTs facilitates the use of 2D array beamforming techniques and shorter wavelengths which in turn allows for more resolution of the obtained 3D images. Integrating on-chip electronics can be made with wafer bonding or flip-chip techniques, or directly fabricating CMUTs and associated electronics on standard CMOS technologies with extra post-processing steps added for sacrificial etching, membrane release and acoustic port sealing [2].

Different fabrication techniques for CMUTs have been proposed over the years [3-5]. The most classical approach consists of an oxide deposition between two polysilicon layers and subsequent sacrificial release of the oxide by immersing the devices in a wet chemical solution which attacks the oxide through the etch holes [6]. A vacuum cavity gap has to be then formed by extracting the air between the membranes and sealing the etch holes. An alternative fabrication technique is the wafer bonding technique [7], in which membranes and cavities are defined on different wafers, which are then bonded in a vacuum environment. This softens the limitations set by having to make vacuum and seal the etch holes in the traditional sacrificial layer release process, and introduces some other advantages such as tight repeatability and control of CMUT properties, at the cost of fabrication complexity.

CMUT arrays are made of small elements of a few tens of micron characteristic length, making up an array typically of 100 to 500 micron length. Different geometries of CMUT elements and CMUT arrays have been proposed including circular, square and hexagonal elements and sparse, square matrix [8] and ring arrays [3]. CMUT array optimization techniques have been proposed in [9]. Desirable performance characteristics for CMUTs include high membrane displacement, low DC bias voltages, reliability, easiness in fabrication and integration with CMOS processes.

At the same time, multi-user MEMS processes (MUMPs) represent low cost standard fabrication methods for MEMS manufacturing which exploit cost and wafer sharing among a number of projects for different users. In particular, the PolyMUMPs process offered by MEMSCAP Inc. [10] offers three polysilicon layers, one gold metallic layer and two sacrificial oxide layers, and constitutes one of the more mature MEMS fabrication process options at this time.

The use and limitations of commercial MUMPs for CMUT fabrication have been reported in $[8,11]$. Amongst the most important practical limitations found were those concerning fixed layer thicknesses, fixed polysilicon doping, maximum allowed die size, and the radiation disturbance caused by etch holes and any other acoustical ports present in the design. However, the added easiness in fabrication and cost- 
effectiveness offered by MUMPs makes this option still promising.

In this paper, the square, circular and hexagonal geometries of CMUT elements are studied through FEM simulations. Results are compared for future design reference.

\section{PHYSICAL PRINCIPLE}

The physical principle underneath the CMUT will be explained in this section, based on the theory developed in [12]. CMUTs are basically parallel plate capacitors with a vacuum gap in the middle. They can be used as transmitters or receivers though a different driving circuit needs to be used in each case. The transmitter driver consists of a pulse generator and a bias tee which sums up the applied AC and DC bias voltages. The receiver driver case consists of a bias tee to sum up $\mathrm{AC}$ and $\mathrm{DC}$, plus an amplifier stage.

As a DC voltage is applied between the terminals, an electrostatic force tending to close the gap develops proportional to the square of the applied bias voltage:

$$
F_{\text {elec }}=\frac{\varepsilon_{0} A V^{2}}{2\left(d_{0}-x\right)^{2}}
$$

where $\varepsilon_{0}$ is the permittivity of free space, $A$ is the area of the plates, $V$ stands for the applied bias voltage between the plates, $d_{0}$ is the initial gap height and $\mathrm{x}$ is the membrane displacement.

At the same time, the moving plate behaves mechanically as a spring, which produces a restoring force proportional to the spring constant:

$$
F_{\text {mech }}=-k x
$$

The minus sign indicates different direction from the electrostatic force, the spring trying to pull upwards. When the sum of the electrical force and the spring force equals zero, the following expression is obtained:

$$
V=\sqrt{\frac{2 k x}{A \varepsilon_{0}}}\left(d_{0}-x\right)
$$

Expression (3) gives a relation between membrane displacement $x$ and applied bias voltage $V$. Pull-in or collapse occurs when $d V / d x=0$. Making the calculation and substituting into (3) the pull-in voltage results as:

$$
V_{\text {pullin }}=\sqrt{\frac{8 k d_{0}^{3}}{27 \varepsilon_{0} A}}
$$

\section{FABRICATION TECHNOLOGY}

MUMPs processes are mainly attractive for cost reasons, besides the confidence that well-established processes may bring for designers. For example, the PolyMUMPs technology is by now well consolidated and is briefly described now as it was the process considered for our CMUT simulations. The fact that this fabrication process has by now a history of approximately 80 runs makes it trustworthy and removes fabrication process failure uncertainty. For further details on this technology the reader is referred to [10].
The process set starts by a $600 \mathrm{~nm}$ silicon nitride layer (acting as insulator) first being deposited on the wafers using low pressure chemical vapor deposition (LPCVD). A $500 \mathrm{~nm}$ polysilicon layer POLY0 acting as a ground electrode is then LPCVD deposited. A $2.0 \mu \mathrm{m}$ LPCVD phosphosilicate glass (PSG) sacrificial layer is then formed, constituting the first sacrificial oxide layer, providing a gap between the isolated substrate and the next polysilicon layer, POLY1, which is subsequently deposited by LPCVD with a thickness of $2.0 \mu \mathrm{m}$. The second sacrificial oxide layer is then formed on top, with a thickness of $0.75 \mu \mathrm{m}$, providing the gap between POLY1 and POLY2. The second structural layer POLY2 is deposited with $1.5 \mu \mathrm{m}$ after the etching of the second oxide layer, followed by a $0.5 \mu \mathrm{m}$ gold layer with a thin adhesion layer that allows for probing, bonding and electrical routing. After deposition and patterning of all the seven layers, a hydrofluoric acid release is performed that etches away the two oxide layers and forms the releasing gaps.

For our FEM simulations, CMUT fabrication was considered between the $0.5 \mu \mathrm{m}$ thick POLY0 and $1.5 \mu \mathrm{m}$ thick POLY2, skipping the $2.0 \mu \mathrm{m}$ thick POLY1 layer. For clarity, the layer stack of simulated CMUT elements is shown in Fig. 1.

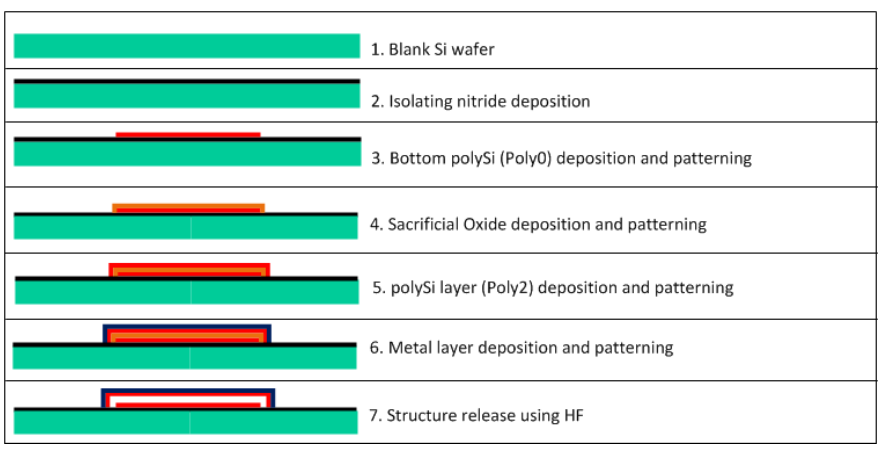

Fig. 1. Simplified CMUT fabrication process layer stack, based on PolyMUMPs, consisting of two polysilicon layers, initially separated by a sacrificial oxide layer, and a top metal conduction layer.
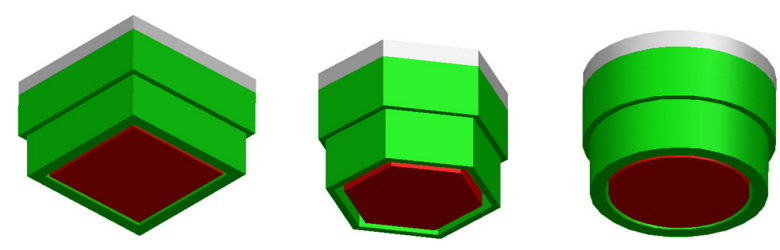

Fig. 2 3D models of the different geometries of the study (z-axis times 50 increase), showing the POLY0 bottom electrode, ANCHOR2 support structure, POLY2 membrane and METAL top electrode.

\section{GEOMETRIES}

The three geometries considered are shown in Fig. 2, showing the layers used in each design (POLY0, POLY2, ANCHOR2, METAL), for the three (square, circular and hexagonal) CMUT element geometries.

Layer POLY0 constitutes the bottom electrode, shown underneath. Layers POLY2 and METAL constitute the vibrating membrane and top electrode, respectively. ANCHOR2 fixes POLY2 to the isolating nitride layer. 
Five CMUTs were considered according to the following considerations: if a constant pitch is desired, areas decrease in the following order: square, circle and hexagon. If a constant membrane area is desired, pitches increase as square, circle and hexagon. Therefore the five elements given in TABLE I were considered. Although some of these dimension values should be varied slightly in order to fully comply with design rules, the proposed models represent a good trade-off between a realistically manufacturable CMUT element and at the same time simple enough to mesh and simulate reasonably fast with FEM / MEMS simulation software.

TABLE I. ELEMENTS CONSIDERED FOR CONSTANT AREA AND CONSTANT PITCH GEOMETRY COMPARISONS.

\begin{tabular}{|l|c|c|}
\hline \multicolumn{1}{|c|}{ Geometry / Property } & Pitch( $\boldsymbol{\mu m})$ & $\begin{array}{c}\text { Membrane } \\
\text { Area }\left(\boldsymbol{\mu m}^{2}\right)\end{array}$ \\
\hline 1. Square & 500.0 & 122500 \\
\hline 2. Circle (constant pitch) & 500.0 & 96211.0 \\
\hline 3. Hexagon (constant pitch) & 500.0 & 79566.0 \\
\hline 4. Circle (constant area) & 564.2 & 122500 \\
\hline 5. Hexagon (constant area) & 620.3 & 122500 \\
\hline
\end{tabular}

\section{FEM MESHING AND BOUNDARY CONDITIONS}

Due to the different geometries, meshing cannot be made exactly identical for each element. Meshing parameters changed from structure to structure including meshing algorithm, number of elements, element aspect ratio, element types and sizes. The FEM modeling of this work was done with the commercial software CoventorWare ${ }^{\mathrm{TM}}$ by Coventor Inc., which includes Designer ${ }^{\mathrm{TM}}$ and Analyzer ${ }^{\mathrm{TM}}$. Boundary conditions applied imply a zero displacement over all transducer borders (ANCHOR2) as well as the bottom electrode, which remains fixed. For all geometries the bottom electrode was meshed with Manhattan brick elements and the ANCHOR2 and POLY2 were meshed either with Manhattan (square geometries) or extruded bricks (circular and hexagonal geometries).

\section{RESULTS}

\section{A. Maximum displacements and mode shapes}

Maximum element displacements were computed with the mechanical solver and are shown in TABLE II. Under both constant area and constant pitch comparisons the circular geometry produced the highest displacement (Fig. 3).

Maximum displacements do change over geometries when a constant pitch is desired, whereas for a constant area comparison displacement variations were found smaller. Still, variations do exist: the highest displacement of $1.9 \mu \mathrm{m}$ is attributed to the circular geometry, decreasing for the hexagon $(1.2 \mu \mathrm{m})$ and square $(0.74 \mu \mathrm{m})$.

A modal mechanical analysis was set producing the first five vibration modes for each CMUT element, which are given in TABLE III, including resonances and antiresonances. As geometries considered had a $500 \mu \mathrm{m}$ characteristic side length, which determines resonance frequency values, obtained numerical values resulted in the tens of $\mathrm{kHz}$ range. Differences amongst geometries are attributed to the inherent geometrical and size changes.

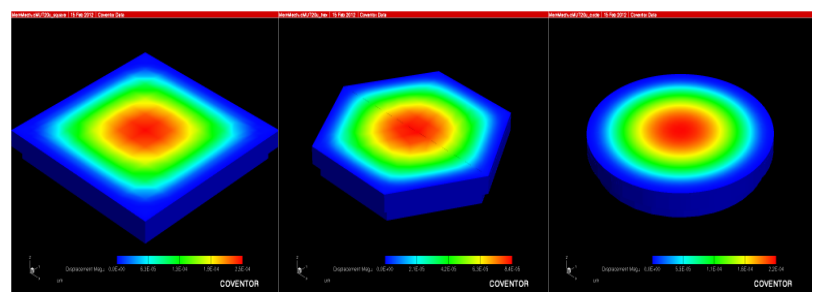

Fig. 3. FEM displacement magnitude distribution for the three geometries considered.

\section{B. Pull-in}

Pull-in occurs as the downward electrostatic force overcomes the upward mechanical spring force (Eqn. 4). On the other hand, as the applied DC voltage is decreased, lift-off would occur as the spring force exceeds the electrostatic force and the membrane stops touching the ground, considered the device was still alive and working.

Pull-in voltages were investigated for the five elements and are given in TABLE II. For this analysis, coarser FEM mesh structures were set in order to keep reasonable computation times. The evolution of $z$ displacement as pull-in is reached for each one of the elements is shown in Fig. 4. For constant area comparison, pull-in values fall within the $12-20 \mathrm{~V}$ range, increasing as circle, hexagon and square. For a constant pitch comparison, the hexagon, which had the lowest area, was the element in which highest pull-in voltage was found.

TABLE II. MAXIMUM DISPLACEMENTS AND PULL-IN VOLTAGES CALCULATED FOR ALL ELEMENTS OF THE STUDY.

\begin{tabular}{|l|c|c|c|c|}
\hline \multicolumn{1}{|c|}{ Geometry / Property } & Pitch $(\boldsymbol{\mu m})$ & $\begin{array}{c}\text { Membrane } \\
\text { Area }(\boldsymbol{\mu m})\end{array}$ & $\begin{array}{c}\text { Maximum } \\
\text { displacement } \\
(\boldsymbol{\mu m})\end{array}$ & $\begin{array}{c}\text { Pull-in } \\
\text { voltage (V) }\end{array}$ \\
\hline 1. Square & 500.0 & 122500 & 0.74 & 19.69 \\
\hline 2. Circle (constant pitch) & 500.0 & 96211.0 & 0.99 & 15.93 \\
\hline 3. Hexagon (constant pitch) & 500.0 & 79566.0 & 0.63 & 21.56 \\
\hline 4. Circle (constant area) & 564.2 & 122500 & 1.9 & 12.19 \\
\hline 5. Hexagon (constant area) & 620.3 & 122500 & 1.2 & 13.44 \\
\hline
\end{tabular}


TABLE III. FEM MODAL Frequencies Found For EACH GEOMETRY $(\mathrm{Hz}) \cdot \mathrm{p}=\operatorname{PITCH}(\mu \mathrm{m}) . \mathrm{A}=\mathrm{ArEA}\left(\mu \mathrm{m}^{2}\right)$.

\begin{tabular}{|c|c|c|c|c|c|}
\hline & 1. Square & 2. Circular & 3. Hexagonal & 4. Circular & 5. Hexagonal \\
\hline MODE NUMBER & $p=500, A=122500$ & $p=500, A=96211$ & $p=500, A=79566$ & $p=564.2, A=122500$ & $p=620.3, A=122500$ \\
\hline 1 & 81528.7 & 75737.6 & 85435.7 & 69445.4 & 71048.9 \\
\hline 2 & 153132.6 & 140623.4 & 170663.1 & 115866.1 & 121547.2 \\
\hline 3 & 153132.6 & 141121.4 & 170670.9 & 115892.9 & 121595.9 \\
\hline 4 & 223918.3 & 226888.5 & 281607.8 & 181646.1 & 191719.1 \\
\hline 5 & 273616.1 & 227240.7 & 281646.4 & 181724.4 & 191862.1 \\
\hline
\end{tabular}

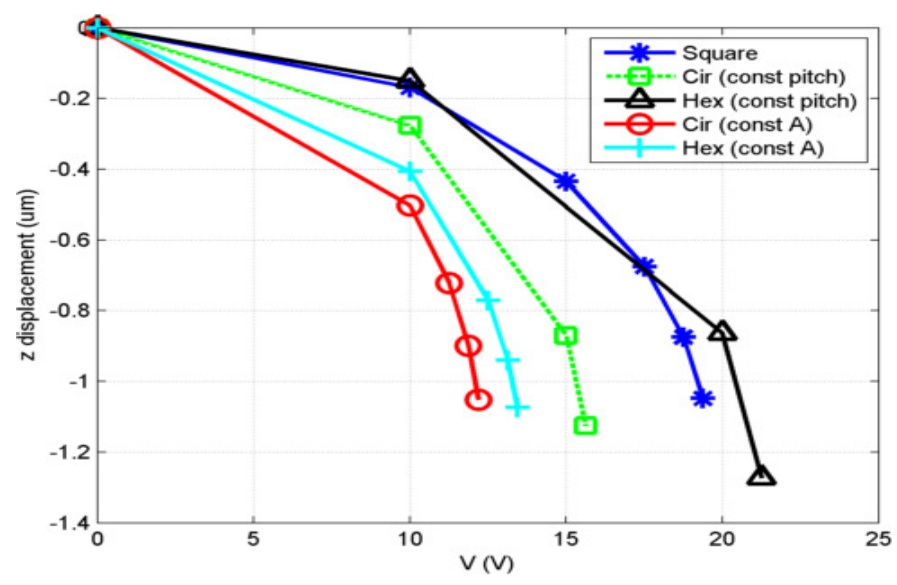

Fig. 4. Evolution of vertical displacement as pull-in voltage is reached for each geometry.

\section{CONCLUSION}

A set of five different CMUT elements with square, circular and hexagonal geometries has been compared. for electromechanical parameter comparison via FEM analyses. For constant area comparison, the circular geometry showed lowest resonance peaks and the square geometry showed highest resonance and lowest pitch. Comparison under constant pitch showed that the circular geometry had lowest resonance and the hexagonal showed highest resonance and lowest area. Amongst the three geometries considered, the circular geometry showed the highest maximum displacement of the membrane. Pull-in voltages were found in the range of 10 to 20 $\mathrm{V}$ and was greatest for the hexagonal geometry. These results may help design considerations and choosing a particular CMUT geometry for a specific task.

\section{ACKNOWLEDGMENT}

Author JML thanks partial funding by the postdoctoral program JAE-DOC granted by the Spanish National Research Council (Consejo Superior de Investigaciones Científicas, C.S.I.C.) through the European Social Fund.

\section{REFERENCES}

[1] O. Oralkan, A. S. Ergun, J. A. Johnson, M. Karaman, U. Demirci, K. Kaviani, T. H. Lee, and B. T. Khuri-Yakub, "Capacitive micromachined ultrasonic transducers: Next-generation arrays for acoustic imaging?," Ieee Transactions on Ultrasonics Ferroelectrics and Frequency Control, vol. 49, pp. 1596-1610, Nov 2002.

[2] C. B. Doody, X. Cheng, C. A. Rich, D. F. Lemmerhirt, and R. D. White, "Modeling and Characterization of CMOS-Fabricated Capacitive Micromachined Ultrasound Transducers," Journal of Microelectromechanical Systems, vol. 20, pp. 104-118, 2011.

[3] B. T. Khuri-Yakub and O. Oralkan, "Capacitive micromachined ultrasonic transducers for medical imaging and therapy," Journal of Micromechanics and Microengineering, vol. 21, May 2011.

[4] E. Cianci, L. Visigalli, V. Foglietti, G. Caliano, and M. Pappalardo, "Improvements towards a reliable fabrication process for cMUT," Microelectronic Engineering, vol. 67-68, pp. 602-608, 2003.

[5] O. Ahrens, A. Buhrdorf, D. Hohlfeld, L. Tebje, and J. Binder, "Fabrication of gap-optimized CMUT," IEEE Transactions on Ultrasonics, Ferroelectrics and Frequency Control, vol. 49, pp. 1321-1329, 2002.

[6] A. S. Erguri, Y. Huang, X. Zhuang, O. Oralkan, G. G. Yarahoglu, and B. T. Khuri-Yakub, "Capacitive micromachined ultrasonic transducers: fabrication technology," IEEE Transactions on Ultrasonics, Ferroelectrics and Frequency Control, vol. 52, pp. 2242 - 2258, 2005.

[7] H. Yongli, A. S. Ergun, E. Haeggstrom, M. H. Badi, and B. T. KhuriYakub, "Fabricating capacitive micromachined ultrasonic transducers with wafer-bonding technology," Microelectromechanical Systems, Journal of, vol. 12, pp. 128-137, 2003.

[8] A. Octavio, R. L. O'Leary, S. M. Whiteley, O. Martinez-Graullera, C. J. Martin-Arguedas, L. Gomez-Ullate, and F. Montero de Espinosa, "Aircoupled linear and sparse cMUT array manufactured using MUMPs process," Microsystem Technologies-Micro-and Nanosystems-Information Storage and Processing Systems, vol. 17, pp. 1635-1644, Oct 2011.

[9] C. Tekes, M. Karaman, and F. L. Degertekin, "Co-array optimization of CMUT arrays for Forward-Looking IVUS," in Ultrasonics Symposium (IUS), 2009 IEEE International, 2009, pp. 1326-1329.

[10 A. Cowen, B. Hardy, R. Mahadevan, and S. Wilcenski, PolyMUMPs Design Handbook. A MUMPs Process, Rev. 13.0: MEMSCAP Inc., 2011.

[11] J. Liu, C. Oakley, and R. Shandas, "Capacitive micromachined ultrasonic transducers using commercial multi-user MUMPs process: Capability and limitations," Ultrasonics, vol. 49, pp. 765-773, 2009.

[12] K. J. V. Vijay K. Varadan, K.A. Jose, RF MEMS and Their Applications: John Wiley \& Sons, Ltd, 2003. 